\title{
A strategic model for network formation
}

\author{
Omid Atabati ${ }^{1 \text { H*}^{*}}$ and Babak Farzad ${ }^{2 \dagger}$
}

\author{
${ }^{*}$ Correspondence: \\ oatabati@ucalgary.ca \\ †Equal contributor \\ ${ }^{1}$ Department of Economics, \\ University of Calgary, 2500 \\ University Drive NW, Calgary, AB \\ T2N 1N4, Canada \\ Full list of author information is \\ available at the end of the article
}

\begin{abstract}
We study the dynamics of a game-theoretic network formation model that yields large-scale small-world networks. So far, mostly stochastic frameworks have been utilized to explain the emergence of these networks. On the other hand, it is natural to seek for game-theoretic network formation models in which links are formed due to strategic behaviors of individuals rather than based on probabilities. Inspired by Even-Dar and Kearns' model (NIPS 19: 385-392, 2007), we consider a more realistic framework in which the cost of establishing each link is dynamically determined during the course of the game. Moreover, players are allowed to put transfer payments on the formation and maintenance of links. Also, they must pay a maintenance cost to sustain their direct links during the game. We show that there is a small diameter of at most four in the general set of equilibrium networks in our model. We achieved an economic mechanism and its dynamic process for individuals which firstly, unlike the earlier model, the outcomes of players' interactions or the equilibrium networks are guaranteed to exist. Furthermore, these networks coincide with the outcome of pairwise Nash equilibrium in network formation. Secondly, it generates large-scale networks that have a rational and strategic microfoundation and demonstrate the main characterization of small degree of separation in real-life social networks. Moreover, we provide a network formation simulation that generates small-world networks.
\end{abstract}

Keywords: Network formation; Linking game with transfer payments; Pairwise stability; Pairwise Nash equilibrium; Small-world phenomenon

\section{Introduction}

In recent years, networks have been extensively studied mostly in terms of their structure but also their formation and dynamics. Structural characteristics of various networks, which emerge from disciplines, such as economics, computer science, sociology, biology, and physics, have been investigated. Many of these networks, in spite of their different origins, indicate large commonalities among their key structural properties, such as small diameter, high clustering coefficient, and heavy-tailed degree distribution which are often quantified by power-law probability distributions. Hence, it is an exciting challenge to study network formation models capable of explaining how and why these structural commonalities both occur and evolve. The series of experiments by Milgram in the 1960s [2] were among the pioneering works that quantified the smallworld phenomenon ${ }^{\mathrm{a}}$ and introduced the 'six degree of separation'. Recent experiments [3] showed that today's online social networks such as Facebook indicate that the degree

(c) 2015 Atabati and Farzad; licensee Springer. This is an Open Access article distributed under the terms of the Creative Commons Attribution License (http://creativecommons.org/licenses/by/4.0), which permits unrestricted use, distribution, and reproduction in any medium, provided the original work is properly credited. 
of separation (for almost any two individuals in a given database) must be even smaller than 4.

The small-world model by Watts and Strogatz [4] was one of the first models that generates networks with small diameter. This work was followed by Kleinberg's stochastic model [5] that was located in a grid graph. It introduced a process that adds links with distance $d$ to the grid with a probability proportional to $1 / d^{\alpha}$. These models, however, can not be applicable when there is a strategical purpose in players' making or losing their connections. In these cases, players, which are represented by vertices, strategically establish and sever their connections to obtain an advantageous position in their social network. Hence, we refer to a class of game-theoretic network formation, also known as strategic network formation (see [6,7] for comprehensive surveys). Models in this class are in their early efforts. They generally assume that players make connections based on a utility maximization and treat the network as the equilibrium result of the strategic interactions among players.

\section{Our contribution}

Our game-theoretic network formation model is mainly inspired by Even-Dar and Kearns (EK model) [1]. In their model, players (i.e., vertices) seek to minimize their collective distances to all other players. The network formation starts from a seed grid. Also, the cost of establishing each link in this model is considered to be the grid distance between the endpoint players of that link and the power of $\alpha$, which is the parameter of the model. Hence, their model uses a fixed link-pricing for each link. Both link creation and link severance are considered unilateral by players. In addition, the equilibrium is defined in terms of link stability: no players benefit from altering a single link in their link decisions. The EK model achieves small diameter link stable networks within the threshold of $\alpha=2$. However, they faced an unbounded diameter that grows with the number of players, when $\alpha>2$.

We define three types of costs for links: (i) the link-price, (ii) the maintenance cost, and (iii) the transfer payment. The link-price $p_{i j}$ is the price of establishing link $i j$. Only the initiator of the connection would bear its payment. It is a one-time charge when establishing the link. We introduce a new viewpoint to this game that better echoes with reality by constructing a dynamic link-pricing. When characterizing the formation of a network, the involved dynamics is a crucial and determining element. We aim to effectuate the impact of this dynamics in our model with the revised link-pricing. We update the used distances of each pair of players in the related link-prices from the current network rather than sticking with the initial grid distances.

In addition, we introduce maintenance costs to make the model more real where a player can give up her payment and sever her connection, if she will be better off by doing so. Also, it is reasonable to assume that refunding the link-prices may not be possible in lots of real-world scenarios. Hence, maintenance costs make the link severance scenario well-defined. In our model, player $i$ is charged for all of its incident links by considering recurring maintenance $\operatorname{costs} c_{i j}$. In other words, for each decision made in the game, players should take the maintenance cost of their incident links into their consideration. Lastly, we allow individuals to put transfer or side payments on their links. Transfers are a sort of communication between players for their connections. In fact, without transfer payments, many agreements on these connections would simply never exist. 
We use the myopic notion of pairwise stability with direct and indirect transfers $\left(P S^{t}\right)^{\mathrm{b}}$ as our equilibrium notion. This notion has the advantage of being compatible with the cooperative and bilateral nature of link formation. Moreover, the pairwise stability has the desirable simplicity required for analyzing players' behaviors under this notion ${ }^{c}$.

On the other hand, due to the bilateral agreement for any link formation, the typical notion of Nash equilibria have some drawbacks in terms of coordination failures, e.g., an empty network is always a Nash equilibrium. In other words, Nash equilibria networks can contain some mutually beneficial link(s) that are left aside. To solve this coordination problem when employing Nash equilibria, the notion of pairwise Nash stability ${ }^{\mathrm{d}}$ was introduced. Pairwise Nash stable $\left(\mathrm{PNS}^{t}\right)$ networks are at the intersection of the set of Nash equilibrium networks and the set of pairwise stable networks.

In this paper, we not only guarantee the existence of pairwise stable networks but also demonstrate that, in our model, the set of pairwise stable networks coincide with the set of pairwise Nash stable networks. Finally, we show that the general set of equilibrium networks exhibits a short diameter of at most 4 . The rest of this paper is organized as follows. In the 'Preliminaries' section, we explain the required preliminaries and provide the setup of our model. 'Fixed link-pricing model' section contains an analysis and extension to the EK model. We then provide the main results for our grid-based model with the dynamic link-pricing and transfer payments in the 'Dynamic link-pricing model with transfer payments' section. In the 'Simulations' section, we present the outcome of a network formation simulation that we carried out.

\section{Preliminaries}

The network and players. Let $N=\{1, \ldots, n\}$ be the set of $n$ players forming a network $G$. Network $G$ is undirected and includes a list of pairs of players who are linked to each other. Link $i j \in G$ indicates that player $i$ and player $j$ are linked in $G$. Let $G^{N}$ denote the complete network. The set $\mathcal{G}=\left\{G \subseteq G^{N}\right\}$ consists of all possible networks on $N$. We define network $G_{0}$ to be the starting network of the game, which is also called the seed network. The set of player i's neighbors in $G$ is $\mathcal{N}_{i}(G)=\{j \mid i j \in G\}$. Similarly, $\mathcal{L}_{i}(G)=$ $\left\{i j \in G \mid j \in \mathcal{N}_{i}(G)\right\}$ denotes the set of links, which are incident with player $i$ in $G$. If $l$ is a subset of $\mathcal{L}_{i}(G)$, then $G-l$ is the network resulted by removing the existing links in the set $l$ from $G$. Similarly, if $l=\left\{i j \mid j \notin \mathcal{N}_{i}(G), j \neq i\right\}$, then the network $G+l$ is obtained by adding the links in set $l$ to $G$.

The utility of network $G$ for player $i$ is given by a function $u_{i}: G \rightarrow \mathbb{R}^{+}$. Let $\mathbf{u}$ denote the vector of utility functions $\mathbf{u}=\left(u_{1}, \ldots, u_{n}\right)$. So, $\mathbf{u}: \mathcal{G} \rightarrow \mathbb{R}^{N}$. Also, the value of a network, $v(G)$, is the summation of all players' utilities in the network $G$, i.e., $v(G)=\sum_{i=1}^{n} u_{i}(G)$. For any network $G$ and any subset $l_{i}(G) \subseteq \mathcal{L}_{i}(G)$, the marginal utility for a player $i$ and a set of links $l_{i}(G)$ is denoted by $m u_{i}\left(G, l_{i}(G)\right)=u_{i}(G)-u_{i}\left(G-l_{i}(G)\right)$.

Strategies, transfer payments. Each player $i \in N$ announces an action vector of transfer payment $\mathbf{t}^{i} \in \mathbb{R}^{n(n-1) / 2}$. The entries in this vector indicate the transfer payment that player $i$ offers (to pay) or demands (to gain) on the link $j k$. If $i \in\{j, k\}$, then we call it a direct transfer payment. Otherwise, it is called an indirect transfer payment. Typically, individuals can make demands (negative transfers) or offers (non-negative transfers) on their direct connections. However, they can only make offers (and not demands) on the indirect transfer payments ${ }^{\mathrm{e}}$. In addition, a link $j k$ is formed if and only if $\sum_{i \in N} t_{j k}^{i} \geq 0$. Thus, the profile of strategies or the announced vectors of transfer payments for all players 
is defined: $\mathbf{t}=\left(\mathbf{t}^{1}, \ldots, \mathbf{t}^{n}\right)$. Consequently, the network $G$, which is formed by this profile of strategies $\mathbf{t}$, can be denoted as follows: $G(\mathbf{t})=\left\{j k \mid \sum_{i \in N} t_{j k}^{i} \geq 0\right.$, where $\left.j, k \in N\right\}$.

The payoff function. The distance between a pair of players $i$ and $j$ in $G$, denoted by $d_{G}(i, j)$, is defined as the length of a shortest path between $i$ and $j$ in G. Similar to the EK model, players seek to minimize their total distances to all players. This benefit would be considered for each player with respect to the network $G$ and links benefit both endpointsf. The link-price is defined to be $p_{i j}=d_{G}(i, j)^{\alpha}$ for $\alpha>0$. The link-price function is non-decreasing and follows Kleinberg's stochastic model. Also, function $c_{i j}$ denotes the maintenance cost for the link $i j$. The utility function of player $i$ is the negative of her total distances and links expenses and is defined as follows:

$$
u_{i}(G(\mathbf{t}))=-\sum_{j \in N} d_{G(\mathbf{t})}(i, j)-\sum_{j \in \mathcal{N}_{i}}\left(p_{i j}+c_{i j}\right)-\sum_{j k \in G(\mathbf{t})} t_{j k}^{i} .
$$

The dynamic process. The following notion is stated from [15] that motivates the desired dynamics for our analysis.

Definition 1. An improving path represents a sequence of changes from one network to another. The changes can emerge when individuals create or sever a single link based on the improvement in the resulting network relative to the current network.

In each round of the game, one player adapts her strategy with respect to the current state of the network. We assume a random meeting mechanism for vertices (randomly choosing a pair of players), but we start with a seed network instead of an empty network $[16,17]$. If two networks $G$ and $G^{\prime}$ differ in exactly one link, they are said to be adjacent networks. Also, if there exists an improving path from $G$ to $G^{\prime}$, then $G^{\prime}$ defeats $G$.

The equilibrium strategies. In every equilibrium profile of strategies $\mathbf{t}^{*}$, there is no excess in the offer of transfer payments. A transfer payment $t_{i j}^{* i}$ is negative if and only if maintaining the existing link $i j$ is not beneficial for $i$. In other words, $i$ 's utility from network $G$ is smaller that her utility from network $G-i j$. We refer to this difference as a utility gap. Player $i$ can only use a transfer payment equal to her utility gap. Hence, for an equilibrium profile of strategies $t_{j k}^{* i}$ that forms equilibrium network $G, G\left(\mathbf{t}^{*}\right)=$ $\left\{j k \mid \sum_{i \in N} t_{j k}^{* i}=0, j, k \in N\right\}$.

We would like to indicate that other generalization of transfers' distribution among players are not among the main focuses of this paper ${ }^{g}$.

The Definitions of equilibrium notions are as follows:

Definition 2. A network $G$ is pairwise stable with transfers $\left(\mathrm{PS}^{t}\right)$ with respect to a profile of utility functions $\mathbf{u}$ and a profile of strategies $\mathbf{t}$ that creates network $G$ if

(a) $i j \in G \Longrightarrow u_{i}(G) \geq u_{i}(G-i j)$ as well as $u_{j}(G) \geq u_{j}(G-i j)$,

(b) $i j \notin G \Longrightarrow u_{i}(G) \geq u_{i}(G+i j)$ as well as $u_{j}(G) \geq u_{j}(G+i j)$.

Also, $\mathrm{PS}^{t}(u)$ denotes the family of pairwise stable networks with transfers.

A pure strategy profile $\mathbf{t}^{*}=\left(\mathbf{t}^{* 1}, \ldots, \mathbf{t}^{* n}\right)$ forms a Nash equilibrium in the linking game with transfers if $u_{i}\left(G\left(\mathbf{t}^{i}, \mathbf{t}^{*-i}\right)\right) \leq u_{i}\left(G\left(\mathbf{t}^{*}\right)\right)$ holds for all $i \in N$ and all $t_{i} \in T_{i}$, where 
$\mathbf{t}_{-i}^{*}$ is the equilibrium strategy for all players other than $i$, and $T_{i}$ is the set of all available strategies for $i$. We can also indicate that in the context of network formation, a network $G$ is Nash stable if $\forall i \in N$ and $\forall l_{i}(G) \subseteq \mathcal{L}_{i}(G): u_{i}(G) \geq u_{i}\left(G-l_{i}(G)\right)$.

Definition 3. A pure strategy profile $\mathbf{t}^{*}=\left(\mathbf{t}^{* 1}, \ldots, \mathbf{t}^{* n}\right)$ forms a pairwise Nash equilibrium in the linking game with transfers if

1. It is a Nash equilibrium, and

2. There does not exist any $i j \notin G\left(\mathbf{t}^{*}\right)$, and $t \in T$ such that

(a) $u_{i}\left(G\left(\mathbf{t}_{i j}^{i}, \mathbf{t}_{i j}^{j}, \mathbf{t}_{-i j}^{*}\right)\right) \geq u_{i}\left(G\left(\mathbf{t}^{*}\right)\right)$,

(b) $u_{j}\left(G\left(\mathbf{t}_{i j}^{i}, \mathbf{t}_{i j}^{j}, \mathbf{t}_{-i j}^{*}\right)\right) \geq u_{j}\left(G\left(\mathbf{t}^{*}\right)\right)$, and

(c) at least one of (1) or (2) holds strictly,

where $\mathbf{t}_{-i j}^{*}$ includes all players' strategies in $\mathbf{t}^{*}$ except player $i$.

A tutorial example. Suppose that Figure 1 shows a sub-network of a network $G$ that is obtained through an improving path. Also, assume that player $i$ considers establishing a link to player $j$ in the next random meeting. For this example, let us assume $\alpha=2$ and $c_{i j}=10$ for all $i$ and $j$. Furthermore, $B_{i}(G+i j, i j)=-\sum_{k \neq i}\left(d_{G+i j}(i, k)-d_{G}(i, k)\right)$ defines the benefit of reduced distances in the whole network $G$ that player $i$ is received after adding link $i j$ to $G$. We assume that $B_{i}(G+i j, i j)=30$ and $B_{j}(G+i j, i j)=5$ in this example.

According to the dynamic link-pricing, $p_{i j}=3^{2}=9$. First, we can verify that player $i$ has an incentive to buy link $i j$, as $B_{i}(G+i j, i j)=30 \geq 9+10=19$. However, there is no advantage for player $j$ in this linking, as $B_{j}(G+i j, i j)=5<10$. Therefore, player $j$ must demand the transfer payment $t_{i j}^{j}=-5$ that makes her indifferent regarding this linkage. Player $i$ can offer the transfer payment $t_{i j}^{i}=10-5=5$ to player $j$, since creating $i j$ is still beneficial for $i$, as $30 \geq 5+19=24$. Consequently, link $i j$ can be added to $G$ and network $G^{\prime}$ is achieved along the improving path of game. The network formation continues until a pairwise stable network with transfers is reached. Note that we can also

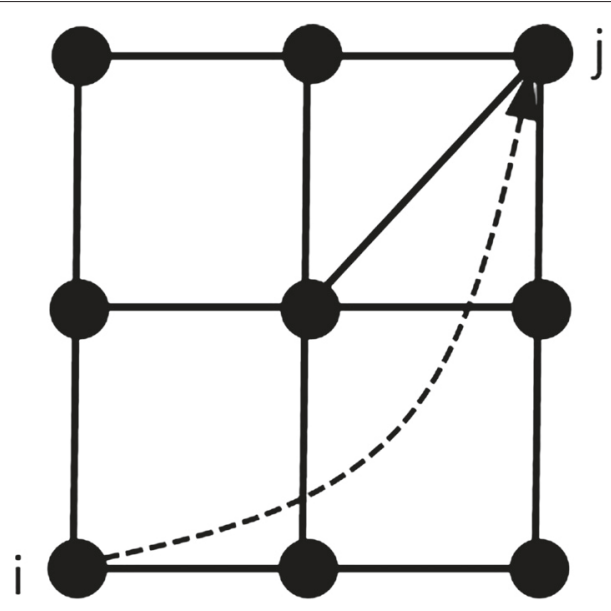

Figure 1 An example of a sub-network from network $G$ during the dynamic process. 
consider the indirect transfers that other players may offer for this linkage, which is not stated in this example for simplicity.

\section{Fixed link-pricing model}

In this section, we study the EK model [1] and consider an extension to this model. This also helps us to provide some insights regarding our results in the 'Dynamic link-pricing model with transfer payments' section.

\section{The presence of cycles}

The EK model takes a $\sqrt{n} \times \sqrt{n}$ grid as its seed network. It defines the link-price $p_{i j}=$ $d_{G_{0}}(i, j)^{\alpha}$ for $\alpha>0$ and defines $d_{G_{0}}(i, j)$ to be the grid distance of $i$ and $j$. Consequently, the link prices are fixed during the course of the game. Furthermore, this model defines the set $\mathbf{s}_{i} \in\{0,1\}^{n-1}$ to be the action set of player $i$ such that $s_{i j}$ is one when player $i$ creates a link to player $j$. Also, each link benefits both endpoints and $s_{i j}=1$ if $s_{j i}=1$. The utility function for player $i \in N$ is $u_{i}(G(\mathbf{s}))=-\sum_{j \neq i} d_{G_{0}}(i, j)-\sum_{j \in \mathcal{N}_{i}} p_{i j}$.

In the EK model, link creation is unilateral. Moreover, creation of a link only requires the agreement of at least one of the endpoint players of the link. This is in contrast to our model in which the presence of each link needs the consent of both players. Also, there is no transfer payment and maintenance cost in this model. Players can receive a refund of the link-prices given the severance of links. This model uses the notion of link stability, where link stable networks are immune against unilateral creation or severance of a single link by each player.

A problem that can arise in this model concerns the fact that the network formation may not converge to a link stable network. In other words, there exists the possibility for the formation of cycles in the evolving networks during this network formation model, as it is defined in the following.

Definition 4. A cycle $C$ is a set of networks $\left(G_{1}, \ldots, G_{k}\right)$ such that for any pair of networks $G_{i}, G_{j} \in C$, there exists an improving path connecting $G_{i}$ to $G_{j}$. In addition, a cycle $C$ is a closed cycle, if for all networks $G \in C$, there does not exist an improving path leading to a network $G^{\prime} \notin C$.

Generally, the presence of negative externalities can be seen as one of the potential reasons in the formation of cycles in linking games. Consider the following grid-based example shown in Figure 2. In this example, we can observe the formation of a cycle in the game.

Assume that $48<3^{\alpha}<49$. First, it is easy to verify that player $s$ has an incentive to create link st. Now, a cycle of strategical updates may be formed as follows. Player $u$ saves 57 in $\sum_{i=1}^{n} d_{G(\mathbf{s})}\left(u, v_{i}\right)$ as it can be verified that the distance to $u$ of nine players in area $i$ is reduced by 1 and the distance to $u$ of 24 players in area $i i$ is reduced by 2 . So, (I) player $u$ has an incentive to buy link $u v$, as $p_{u v}=d_{G_{0}}(u, v)^{\alpha}=3^{\alpha}<9+48=57$. Then, with similar observations, it can be seen that the following strategical changes will be made in this order. (II) Player $w$ buys link $w u$ as $p_{w u}=d_{G_{0}}(w, u)^{\alpha}=3^{\alpha}<49$. (III) Player $u$ is no longer willing to maintain link $u v$, as with existing link $w u$, it has a benefit of only 48. Therefore, $u$ returns the link $u v$. (IV) Player $w$ has no incentive to retain link $w u$, as with the removal of link $u v$, it has a benefit of only 34 . So, $w$ returns the 


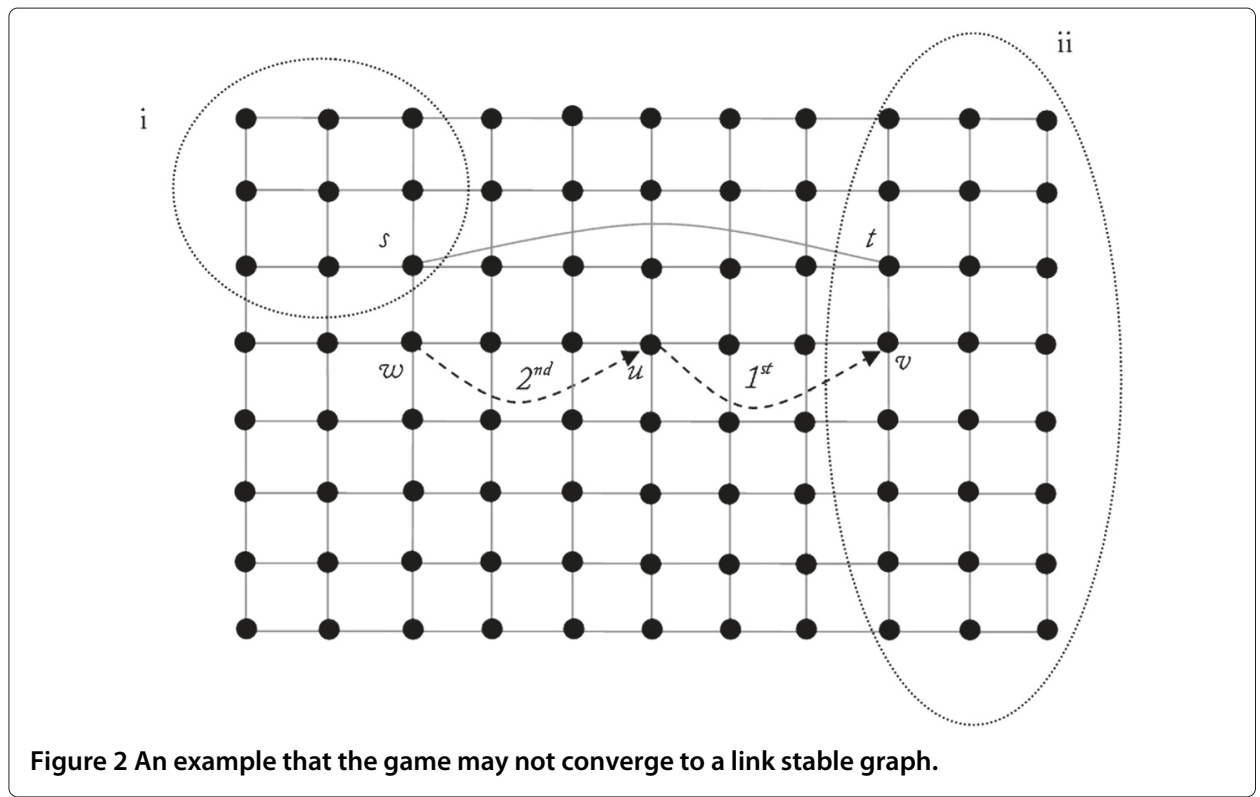

link wu. Thus, a cycle of steps (I) to (IV) may be formed and the game does not converge to stability. The example can be expanded to a large-scale grid as well. We note that player $w$, by establishing $w u$, creates a negative externality for $u$. Since it causes a reduction in player $u$ 's utility, $u$ decides to sever $u v$ that in overall leads to the formation of a cycle.

\section{Forbidding link severance}

In this model, players should be allowed to sever only those links that they themselves have purchased. However, this issue is not clear in the notion of link stability ${ }^{\mathrm{h}}$. Let assume an extension of the EK model with forbidding severing links. Forbidding players to sever their links, although limits the applicability of model, makes the convergence of equilibrium networks possible for the network formation.

Proposition 1. Under the assumption of forbidding link severance in the EK model, the convergence of network formation to link stability is guaranteed.

Proof. When there is no link severance, the existence of negative externalities for players is ruled out. In other words, there is no player whose utility can be hurt during the game. Thus, the total value of the network is increased by each change during the dynamic process. This points to the exact pairwise monotonicity, introduced by Jackson and Watts [15], which guarantees the existence of stable networks. The proof of Theorem 1 can be adapted to imply Proposition 1 .

\section{Dynamic link-pricing model with transfer payments}

\section{Existence of pairwise stable network with transfers}

In all game-theoretic problems, one of the primary questions concerns the existence of equilibria or stable states. This question in the framework of network formation is translated to the existence of pairwise stable networks and have been first addressed by Jackson 
and Watts [15]. We show that their arguments can be extended and adapted in our model. As a result, we guarantee the existence of pairwise stable network with transfers in our model.

While improving paths that start from a seed network may end in an equilibrium network, it is also possible to find the formation of cycles as the result of an improving path. Jackson and Watts showed that in any network formation model there exists either a pairwise stable network or a closed cycle. Their argument is based on the fact that a network is pairwise stable if and only if it does not lie on an improving path to any other network. We provide the following lemma and refer to the work of Jackson and Watts [15] for its proof, where the exact arguments can be applied for the notion of $P S^{t}$ inour model.

Lemma 1. In the network formation model with transfer payments, there exists either an equilibrium network from $\operatorname{PS}^{t}(u)$ or a closed cycle of networks.

Theorem 1. In the linking game with direct and indirect transfers given the utility function in (1),

(a) There are no cycles,

(b) There exists at least one pairwise stable network $\left(\operatorname{PS}^{t}(u)\right)$.

Proof. We can rule out the existence of cycles in a network formation model if we show that the following holds: for any two networks $G$ and $G^{\prime}, G^{\prime}$ defeats $G$ if and only if $v\left(G^{\prime}\right)>$ $\nu(G)$ and $G$ and $G^{\prime}$ are adjacent ${ }^{\mathrm{i}}$. We can briefly argue that our linking game satisfies this condition. Since the direct and indirect transfer payments between players prevent the situations, where a player's utility can get hurt by actions (link addition or deletion) of others. In fact, this is one of the main function of transfers. Therefore, the value of networks through each improving path must be increased. Conversely, if $G$ and $G^{\prime}$ are adjacent in an improving path such that $v\left(G^{\prime}\right)>v(G), G^{\prime}$ must defeat $G$, where $G$ is a network in the cycle.

Now, since there are finitely many networks that can be reached through the dynamic process, if there is a cycle, then the exact pairwise monotonicity of our linking game implies $v(G)>v(G)$; contradiction. Ruling out the existence of cycles along with Lemma 1 guarantees the existence of at least one pairwise stable network with transfer payments.

\section{Strictly pairwise stability}

Now, we show that given the utility function $u($.$) in (1), the family of networks in \mathrm{PS}^{t}(u)$ satisfies the notion of strictly pairwise stability. It is first described by Chakrabarti and Gilles [20], which is a variation of pairwise stability.

Definition 5. A network $G$ is strictly pairwise stable for $\mathbf{u}$ if

(a) $\forall i \in N$ and $\forall l_{i}(G) \subseteq \mathcal{L}_{i}(G), u_{i}(G) \geq u_{i}\left(G-l_{i}(G)\right.$,

(a) $\quad \forall i \in N, i j \notin G$ implies $u_{i}(G+i j)<u_{i}(G)$ as well as $u_{j}(G+i j)<u_{j}(G)$.

In order to progress our argument, we need to provide the following definition and lemma. 
Definition 6. Let $\alpha \geq 0$. A utility function $u($.) is $\alpha$-submodular in own current links on $\mathcal{A} \subseteq \mathcal{G}$ if $\forall i \in N, G \in \mathcal{A}$, and $l_{i}(G) \subseteq \mathcal{L}_{i}(G)$, it holds that $m u_{i}\left(G, l_{i}(G)\right) \geq$ $\alpha \sum_{i j \in l_{i}(G)} m u_{i}(G, i j)$.

The case $\alpha=1$ corresponds to submodularity, also called superadditivity in [9].

Lemma 2. The utility defined in (1) is submodular in own current links.

Proof. The proof is inspired by the arguments in [18]. First, we show the related inequality in Definition 6 holds for the case when the subset $l_{i}(G)$ consists of two distinct links $i j$ and $i k$, which is indicated in the below inequality:

$$
m u_{i}(G, i j+i k) \geq m u_{i}(G, i j)+m u_{i}(G, i k)
$$

If we consider any player such as $m$ in network $G$, the distance between $i$ and $m$ $\left(d_{G}(i, m)\right)$ contributes to the distance expenses in $i$ 's utility. It is important to note that removing any link such as $i j$ or $i k$ from the network $G$ cannot decrease this distance; however, if the removed link belongs to the shortest path between $i$ and $m$ in $G$, then the distance would be increased. This argument can be extended to the severance of two links such as $i j$ and $i k$ from $G$.

$$
\begin{aligned}
& d_{G}(i, m) \leq d_{G-i j}(i, m) \leq d_{G-i j-i k}(i, m) \\
& d_{G}(i, m) \leq d_{G-i k}(i, m) \leq d_{G-i j-i k}(i, m)
\end{aligned}
$$

In computing the marginal utilities of networks $G-i k, G-i j$, and $G-i j-i k$, we should note that the link-prices of removed links cannot be refunded for player $i$ :

$$
\begin{gathered}
m u_{i}(G, i j)=-\sum_{m \neq i}\left(d_{G}(i, m)-d_{G-i j}(i, m)\right)-c_{i j}-t_{i j}^{i} \\
m u_{i}(G, i k)=-\sum_{m \neq i}\left(d_{G}(i, m)-d_{G-i k}(i, m)\right)-c_{i k}-t_{i k}^{i} \\
m u_{i}(G, i j+i k)=-\sum_{m \neq i}\left(d_{G}(i, m)-d_{G-i j-i k}(i, m)\right)-c_{i j}-c_{i k}-t_{i j}^{i}-t_{i k}^{i}
\end{gathered}
$$

According to Inequalities (3) and (4), we can simply imply the Inequality (2). Finally, we can easily extend this argument for any subset of links $l_{i}(G)$.

Proposition 2. Given the utility functions $u($.$) defined in (1), \operatorname{PS}^{t}(u)=P^{\star}(u)$.

Proof. According to the definitions, it can be derived that $P^{\star}(u) \subseteq \operatorname{PS}^{t}(u)$. We further prove that $\operatorname{PS}^{t}(u) \subseteq P^{\star}(u)$.

Let $G \in \operatorname{PS}^{t}(u)$, then for any link $i j \notin G$, neither player $i$ nor $j$ can benefit from creating link $i j$. This is one of the impact of allowing players to put transfer payments on the links. Thus, pairwise stable networks with transfers satisfy the second condition in the Definition 5. Further, we know that $\forall i \in N$ and $\forall j \in l_{i}(G), u_{i}(G-i j) \leq u_{i}(G)$. Let assume 
there are $k$ links in the subset $l_{i}(G)$. Hence, $\sum_{i j \in l_{i}(G)} u_{i}(G-i j) \leq(k) u_{i}(G)$. On the other hand, based on Lemma $2, \sum_{i j \in l_{i}(G)} m u_{i}(G, i j) \leq m u_{i}\left(G, l_{i}(G)\right)$. This implies:

$$
(k) u_{i}(G)-\sum_{i j \in l_{i}(G)} u_{i}(G-i j) \leq u_{i}(G)-u_{i}\left(G-l_{i}(G)\right) .
$$

Since the left-hand side of Inequality (8) is positive, the expression in the right-hand side must be positive too. So, this proves the first condition in the Definition 5 for the networks in $\mathrm{PS}^{t}(G)$.

\section{Convergence to pairwise Nash stability}

Calvó-Armengol and Ilkiliç [13] show the equivalency of pairwise stable networks and pairwise Nash stable networks, given a utility function that is $\alpha$-submodular. It targets the simple observation that given a $\alpha$-submodular utility function, if a player does not benefit from severing any single link, then she does not benefit from cutting any subset of links simultaneously as well. A similar argument can be adapted to our linking game with transfers as well. So, we provide the following proposition without proof.

Proposition 3. Given a profile of utility functions $\boldsymbol{u}$ in (1) in a linking game with transfers, $\mathrm{PS}^{t}(u)=\operatorname{PNS}^{t}(u)$.

\section{Small diameter in equilibrium networks}

We take a large-scale $\sqrt{n} \times \sqrt{n}$ grid as the seed network in this model. In order to prove the main result for the diameter of the equilibrium networks, we provide the following lemmas.

Let $T_{G(\mathbf{t})}(i, j)$ be the set of players that use link $i j$ in their unique shortest paths to $i$ in the network $G(\mathbf{t}): T_{G(\mathbf{t})}(i, j)=\left\{k \in N \mid d_{G^{\prime}(\mathbf{t})}(i, k)>d_{G(\mathbf{t})}(i, k)\right\}$, where $G^{\prime}=G-i j$.

Lemma 3. Let $G(\boldsymbol{t})$ be an equilibrium network and $i, j \in N$ be an arbitrary pair of players in this network. If $i j \notin G(\boldsymbol{t})$, then $\left|T_{G(\boldsymbol{t})}(i, j)\right|<\frac{d_{G(\boldsymbol{t})}(i, j)^{\alpha}+c_{i j}+t_{i j}^{i}}{d_{G(\boldsymbol{t})}(i, j)-1}$.

Proof. Since $i$ and $j$ are not linked in the equilibrium network, the benefit of establishing $i j$ has to be less than its linking costs for $i$ and $j$. On the other hand, $T_{G(\mathbf{t})}(i, j)$ represents the set of players that creates a part of this benefit by reducing the distance $d_{G(\mathbf{t})}(i, j)$ between $i$ and $j$ to 1 . Hence, we can state that paying $d_{G(\mathbf{t})}(i, j)^{\alpha}+c_{i j}+t_{i j}^{i}$, which is necessary for establishing $i j$, cannot be beneficial for player $i$. As a result, $\left|T_{G(\mathbf{t})}(i, j)\right|\left(d_{G(\mathbf{t})}(i, j)-\right)<$ $d_{G(\mathbf{t})}(i, j)^{\alpha}+c_{i j}+t_{i j}^{i}$.

Remark 1. For any $i, j \in N, c_{j i}$ can be noted as an upper bound for the transfer payment $t_{i j}^{i}$. Hence, if $c=\max _{\forall i, j \in N}\left(c_{j k}\right)$, it is an upper bound for any direct transfer payment in the network.

Lemma 4. In any equilibrium network $G(\boldsymbol{t})$, for any player $i \in N$,

let $S_{i}^{d}=\left\{k \in N \mid d_{G(t)}(i, k) \leq d\right\}$. Then, $\left|S_{i}^{d}\right|\left(1+\frac{d^{\alpha}+2 c}{d-1}\right) \geq n$, where $c=\max _{\forall i, j \in N}\left(c_{i j}\right)$. 
Proof. The set $S_{i}^{d}$ consists of players in the neighborhood of $i$ within a distance at most $d$. Furthermore, for each of these players such as $k$ in the set $S_{i}^{d}$, according to Lemma 3, we consider the set $T_{G(\mathbf{t})}(i, k)$. All players outside of this set should use one of players such as $k$ in their shortest path to $i$. As a result, we can cover all players outside the set $S_{i}^{d}$ by allocating a set $T_{G(\mathbf{t})}(i, k)$ to $i$ for all players in set $S_{i}^{d}$. By doing so, an upper bound of $\left|T_{G(\mathbf{t})}(i, k)\right|\left|S_{i}^{d}\right|+\left|S_{i}^{d}\right|$ for the number players in network $(n)$ is achieved.

In order to obtain an upper bound for the set $T_{G(\mathbf{t})}(i, k)$ in wide range of different possible choices for $i$ and $k$, we define $c$ to be the maximum maintenance cost for all possible links in network. According to Remark 1, this is an upper bound for all the possible direct transfer payments as well. Hence, $\left|T_{G(\mathbf{t})}(i, k)\right| \leq \frac{d^{\alpha}+2 c}{d-1}$. By substituting the upper bounds of $T_{G(\mathbf{t})}(i, k)$ and $S_{i}^{d}$ in $\left|T_{G(\mathbf{t})}(i, k)\right|\left|S_{i}^{d}\right|+\left|S_{i}^{d}\right| \geq n$, the desired inequality can be achieved.

Lemma 5 shows an upper bound for the set $\left|S_{i}^{2}\right|$.

Lemma 5. $\left|S_{i}^{2}\right| \leq \Delta^{\alpha}+2 c / k\left(\Delta-\left(h_{1}+h_{2}\left(g_{1}+2\right)+h_{3}\left(2 f_{1}+f_{2}+3\right)\right)\right)$, where $\Delta$ is the diameter of any equilibrium network $G(\boldsymbol{t})$, and $0 \leq k, f_{i}, g_{i}, h_{i} \leq 1$ denote some fractions of players in the set $S_{i}^{2}$ based on their reduced distances to player $i$ when forming the link $i j$. Also, $f_{1}+f_{2}+f_{3}=g_{1}+g_{2}=h_{1}+h_{2}+h_{3}=1$.

Proof. Let $G$ be an arbitrary instance from the set of equilibrium networks in our model, which are the set of pairwise stable networks with transfer $\left(G \in \mathrm{PS}^{t}(u)\right)$, given the utility function $u($.$) in (1). Also, let \mathbf{t}$ be the the profile of strategies for players that forms $G$. Further, assume that the largest distance between any two players (or diameter) in network $G$ exists between two players $i$ and $j$. We denote $\Delta$ to be the size this distance. Note that the pair of $i$ and $j$ is not necessarily unique.

Based on the stable state, we can imply that creation $i j$ is not beneficial for neither $i$ nor $j$. If $j$ wants to establish a link to $i$, $\left|S_{i}^{2}\right|$ is a lower bound for the $j$ 's benefit that comes from the reduced distances to players in $S_{i}^{2}$. This set includes $i$ itself and two subsets of players that are in distance 1 (type 1) and 2 (type 2) from $i$. First, let $k$ represents players in $S_{i}^{2}$ such that their distances to $j$ can be reduced by adding $i j$, as a fraction with respect to all players in $\left|S_{i}^{2}\right|$. Moreover, let $h_{1}$ represents player $i$ itself as a fraction with respect to all players in $\left|S_{i}^{2}\right|$. By establishing $i j$, $j$ 's distance to $i$ reduced by $\Delta-1$.

Furthermore, let $h_{2}$ and $h_{3}$ represent the fractions of the number of type 1 players and type 2 players, respectively, in $S_{i}^{2}$. Their reduced distances for $j$ is computed according to the initial distances of these two types of players in $S_{i}^{2}$ from $j$. Among the type 1 players, there are two subsets of players that $g_{1}$ and $g_{2}$ are their fractions with distance of $\Delta-1$ and $\Delta$ from $j$, respectively. Furthermore, in type 2 players, there are three subsets of players in terms of their distance from $j$ with fractions of $f_{1}, f_{2}$, and $f_{3}$ that are in distance of $\Delta-2, \Delta-1$, and $\Delta$ from $j$, respectively.

Theorem 2. For a sufficiently large network, there is a small diameter of at most 4 for any equilibrium network in the dynamic link-pricing model with transfer payments.

Proof. Based on our arguments in Lemma 4 and Lemma 5, we can state that

$$
n \leq\left(1+2^{\alpha}+2 c\right)\left(\Delta^{\alpha}+2 c\right) / k\left(\Delta-\left(h_{1}+h_{2}\left(g_{1}+2\right)+h_{3}\left(2 f_{1}+f_{2}+3\right)\right)\right) .
$$


For sufficiently large network, when the diameter is greater than $\left\lfloor h_{1}+h_{2}\left(g_{1}+2\right)+\right.$ $\left.h_{3}\left(2 f_{1}+f_{2}+3\right)\right\rfloor$, it contradicts Inequality (9). Clearly, we can specify that $3 \leq 2 f_{1}+f_{2}+3 \leq$ 5 and $2 \leq g_{1}+2 \leq 3$. Thus in this case, the upper bound for the diameter is the weighted average of $1,2 f_{1}+f_{2}+3$, and $g_{1}+2$, and it is surely smaller than 5 . Therefore, the diameter cannot be bigger than 4 for any choice of parameters. However, we cannot have the same claim for smaller diameter and rule out their possibility.

\section{Simulations}

We carried out a set of simulations that improves the EK model by implementing the dynamic link-pricing and a fixed maintenance cost $c$. These simulations generate networks that show (i) a small diameter of at most 4, (ii) a high clustering coefficient (with respect to edge density), and (iii) a power-law degree distribution. The dynamical simulations are implemented on a grid with $n \approx 1,000$. At each iteration of the dynamic process, two players $i$ and $j$ are chosen uniformly at random. Then, with probability $1 / 2$ player $i$ considers establishing a link to $j$ (if $i j \notin G$ ) and with probability $1 / 2$, she considers severing her link to $j$ (if $i j \in G$ ). Note that these considerations are such that in each random meeting, the decision for adding (or removing) a link is implemented based on the corresponded benefit and cost to that link with respect to the current state of the evolved network. We used the notion of link stability. In this set of simulations, we aim to indicate our improvements and extension on the EK model in order to generate small-world networks. Note that by using the dynamic link-prices, the emergence of a small diameter of at most 4 in link stable networks are directly implied similarly by our argument in the 'Small diameter in equilibrium networks' section'.

In many instances of our simulations, it can be seen as in Figure 3 that the degree distribution is a good estimation for the power-law degree distributions in the real-life social networks. Figure 3 shows the impact of parameters $c$ and $\alpha$ on the degree distributions of resulting networks. The larger plots are the distributions where their vertical axis is the probability for degrees and their horizontal axis determines different values for the degree of nodes. The smaller plots are the log-log plots of these distributions. Their vertical axis are the logarithm of the number or the frequency for nodes with different values
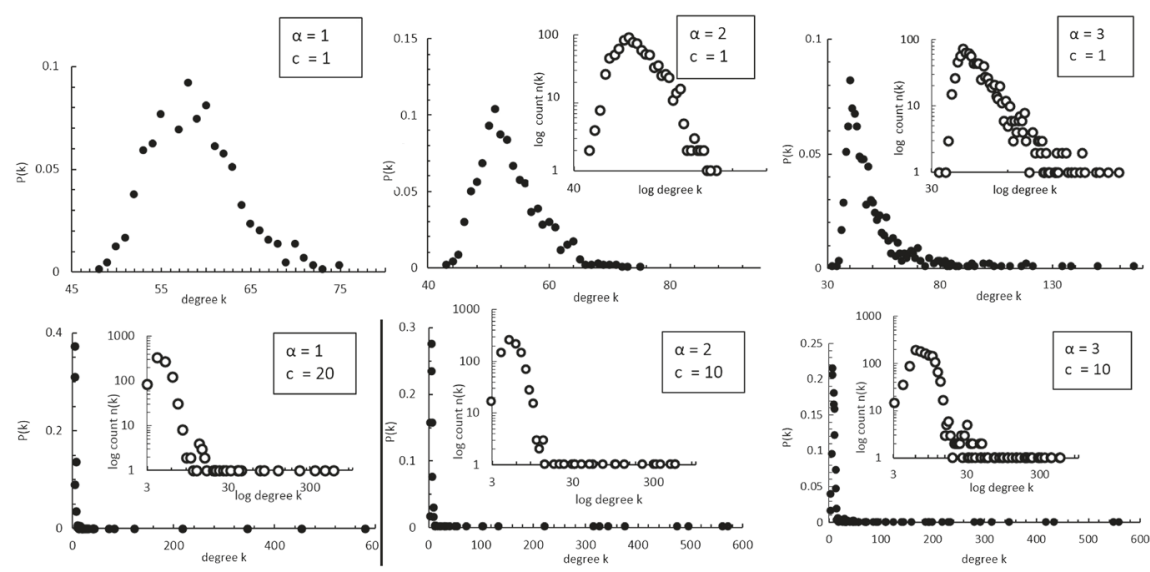

Figure 3 Degree distributions. Structural properties of generated networks in the simulations. 


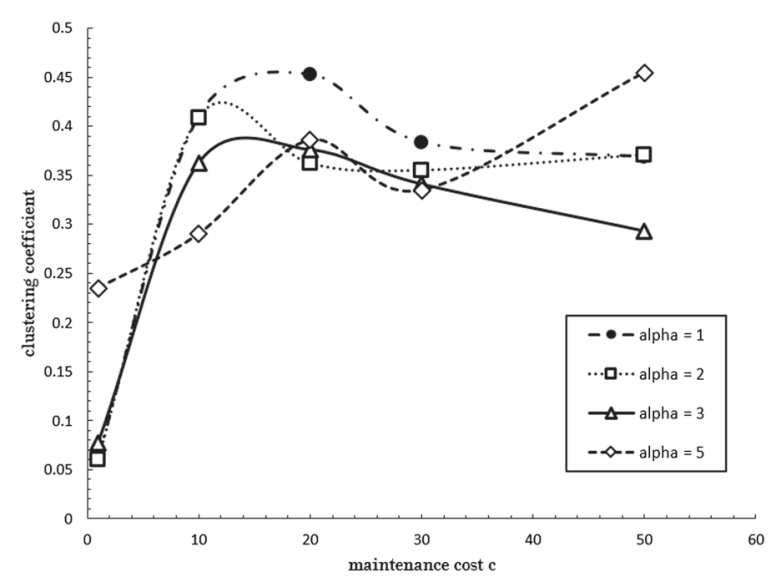

Figure 4 Average clustering coefficients. Structural properties of generated networks in the simulations.

for their degree. Moreover, the appearance of few high degree nodes represents the few hubs in these networks.

Figure 4 demonstrates the clustered structure of the link stable networks: a high average clustering coefficient is present in all instances after increasing the maintenance cost from $c=1$. The high clustering in these networks can be highlighted by pointing out their small edge-density in the range from 0.007 for the network with $c=50, \alpha=5$ to 0.069 for the network with $c=1$ and $\alpha=1$. The diameter in all instances was either 3 or 4 as expected.

\section{Endnotes}

${ }^{\mathrm{a}}$ The principle that individuals are all linked by short chains of connections and acquaintances.

${ }^{\mathrm{b}}$ The pairwise stability is the major notion of stability that assumes myopic players and has been studied in related literature. In a linking game with transfers, it was first introduced as an extension in [8] and then developed in $[9,10]$.

${ }^{\mathrm{C}}$ Computing the best responses of players in Nash equilibria within some similar models $[11,12]$ are proved to be NP-hard.

dSee $[9,10,13,14]$.

e This assumption is reasonable in our framework, since the formation of other links cannot hurt the utility of non-involved players with respect to the distance-based structure of our utility function in (1).

${ }^{\mathrm{f}}$ See e.g. $[8,10,12]$ for some application instances of distance-based payoff structures.

${ }^{g}$ See $[18,19]$ for some instances of study in the case of bargaining between players on network. In fact, despite the rich literature in general for bargaining between players, bargaining on networks is in its early attempts.

${ }^{\mathrm{h}}$ Adding a charging scheme for the maintenance of existing links is a reasonable extension that can resolve this issue, and it is studied in our model.

iThis condition is denoted as exact pairwise monotonicity by Jackson and Watts.

jNote that although the existence of stable networks and convergence to the Nash outcomes would not be guaranteed in this assumption, we achieved a set of link stable networks by implementing many trials for different sets of $\alpha$ and $c$. 
Authors' contributions

OA contributed to the characterization of the model and theoretical arguments, and implemented the simulation. BF contributed to the formulation of the problem and the theoretical arguments. Both authors participated in the organization of this research, and read and approved the final manuscript.

\section{Author details}

${ }^{1}$ Department of Economics, University of Calgary, 2500 University Drive NW, Calgary, AB T2N 1N4, Canada. ${ }^{2}$ Department of Mathematics, Brock University, 500 Glenridge Ave., St. Catharines, ON L2S 3A1, Canada.

Received: 24 October 2014 Accepted: 20 December 2014

Published online: 15 February 2015

\section{References}

1. Even-Dar E, Kearns M. A small world threshold for economic network formation. In: Advances in Neural Information Processing Systems 19. Cambridge, MA: MIT Press; 2007. p. 385-392.

2. Milgram S. The small world problem. Psychol. Today 1967;1:61-67.

3. Daraghmi EY, Yuan S. We are so close, less than 4 degrees separating you and me! Comput. Hum. Behav 2014;30: 273-285.

4. Watts D, Strogatz S. Collective dynamics of small-world networks. Nature 1998;393:440-442.

5. Kleinberg J. The small-world phenomenon: an algorithmic perspective. Symposium on the Theory of Computing. New York: Association of Computing Machinery; 2000, pp. 163-170.

6. De Martí J, Zenou Y. Social networks. Handbook of Philosophy of Social Science. London: SAGE Publications; 2011, pp. 339-361

7. Jackson MO. Social and Economic Networks. Princeton, NJ: Princeton University Press; 2008

8. Jackson MO, Wolinsky A. A strategic model of social and economic networks. J. Econ. Theory 1996;71:44-74.

9. Bloch F, Jackson MO. Definitions of equilibrium in network formation games. Int. J. Game Theory 2006;34(3):305-318

10. Bloch F, Jackson MO. The formation of networks with transfers among players. J. Econ. Theory 2007;133(1):83-110.

11. Myerson R. Game Theory: Analysis of Conflict. Cambridge, MA: Harvard University Press; 1991.

12. Fabrikant A, Luthra A, Maneva EN, Papadimitriou C. H, Shenker S. On a network creation game. In: 22nd Annual ACM Symposium on Principles of Distributed Computing. New York: ACM Press; 2003. p. 347-351.

13. Calvó-Armengol A, Ilkiliç R. Pairwise-stability and Nash equilibria in network formation. Int. J. Game Theory 2009;38(1):51-79.

14. Hellman T. On the existence and uniqueness of pairwise stable networks. Int. J. Game Theory 2012:42:211-237.

15. Jackson MO, Watts A. The existence of pairwise stable networks. Seoul J. Econ 2001;14(3):299-321.

16. Watts A. A dynamic model of network formation. Games Econ. Behav 2001;34:331-341.

17. Jackson MO, Watts A. The evolution of social and economic networks. J. Econ. Theory 2002;106:265-295.

18. Gallo E. Essays in the economics of networks. PhD thesis, University of Oxford: Department of Economics; 2011

19. Bayati M, Borgs C, Chayes J, Kanoria Y, Montanari A. Bargaining dynamics in exchange networks. Working Paper 2011.

20. Chakrabarti S, Gilles R. Network potentials. Rev. Econom. Des 2007;11(1):13-52.

\section{Submit your manuscript to a SpringerOpen ${ }^{\mathcal{D}}$ journal and benefit from:}

$\rightarrow$ Convenient online submission

$\rightarrow$ Rigorous peer review

- Immediate publication on acceptance

Open access: articles freely available online

- High visibility within the field

Retaining the copyright to your article

Submit your next manuscript at $>$ springeropen.com 